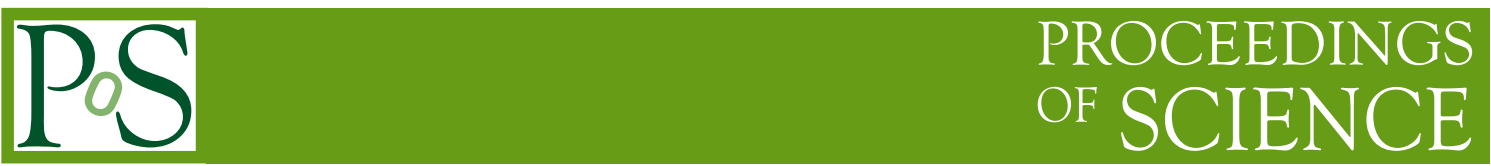

\title{
K-pi scattering lengths at physical kinematics
}

\section{Tadeusz Janowski*}

School of Physics and Astronomy, University of Southampton, Highfield, SO17 1BJ

Southampton, $U K$

E-mail: tjlg11esoton.ac.uk

\section{Peter Boyle}

School of Physics and Astronomy, University of Edinburgh,EH9 3JZ Edinburgh, UK

E-mail: paboyle@ph.ed.ac.uk

\section{Andreas Jüttner}

School of Physics and Astronomy, University of Southampton, Highfield, SO17 1BJ

Southampton, UK

E-mail: juettner@soton.ac.uk

\section{Christopher T. Sachrajda}

School of Physics and Astronomy, University of Southampton, Highfield, SO17 1BJ

Southampton, UK

E-mail: ctsasoton.ac.uk

We present preliminary results on K-pi scattering lengths obtained by the RBC-UKQCD collaboration. The results are obtained using one of our domain wall fermion ensembles with physical quark masses. We demonstrate that the physical point analysis contains large "around-the-world" effects and show techniques that we use to keep these effects under control. Our current estimates for scattering lengths are $m_{\pi} a_{0}=-0.0674(33)$ for $\mathrm{I}=3 / 2$ scattering and $0.1562(50)$ for $\mathrm{I}=1 / 2$, where the errors are statistical only. These results agree with the previous lattice calculations.

The 32nd International Symposium on Lattice Field Theory

23-28 June, 2014

Columbia University New York, $N Y$

\footnotetext{
* Speaker.
} 


\section{Introduction}

Scattering phase shifts are quantities which encode the basic interaction properties of two hadron systems. They can be calculated from first principles using lattice QCD by applying Lüscher's quantization condition [1]. The phase shift will depend on the centre-of-mass energy of the system in a non-trivial way, however if we are interested in low energy scattering processes, it is useful to parametrise it in terms of the scattering length. In this project, we investigate the scattering lengths in the K- $\pi$ system. There are two such processes, depending on the total isospin of the K- $\pi$ system, which can be equal to $1 / 2$ or $3 / 2$. Previous studies of K- $\pi$ scattering lengths have been done with heavy kaon and pion masses and relied on chiral perturbation theory to extrapolate to the physical point [2-5]. This introduces an error due to chiral extrapolation, which is especially large in the $\mathrm{I}=1 / 2$ case. We present below the first calculation done directly at the physical point using 88 configurations of $48^{3} \times 96 \times 242+1$ flavour Iwasaki ensembles with Möbius domain wall fermion action. We choose the bare quark masses to be $a m_{l}=0.00078$ for the light quarks and $a m_{s}=0.0362$ for strange quarks with $\beta=2.13$, which corresponds to the inverse lattice spacing $a^{-1}=1.730(4) \mathrm{GeV}$ [8]. We use $Z_{2} \times Z_{2}$ stochastic sources with periodic boundary conditions in all spatial directions and antiperiodic in time direction. These values correspond to physical pion and kaon masses.

\section{Methodology}

We follow the procedure described in [5]. From Lüscher's quantization condition [1] we have the following dependence of the s-wave phase shift $\left(\delta_{0}\right)$ on the lattice kinematics:

$$
\left(\frac{\tan \delta_{0}(p)}{p}\right)^{-1}=\frac{2}{\sqrt{\pi} L} Z_{00}\left(1, \frac{L^{2}}{4 \pi^{2}} p^{2}\right)
$$

where the 'scattering momentum' $p$ is defined by:

$$
E_{K \pi}=\sqrt{p^{2}+m_{\pi}^{2}}+\sqrt{p^{2}+m_{K}^{2}}
$$

and $Z_{00}(l ; m)$ is defined by:

$$
Z_{00}(l ; m) \equiv \sqrt{4 \pi} \sum_{n \in \mathbb{Z}}\left(n^{2}-m\right)^{-l} .
$$

For $l=1$ the function $Z_{00}$ has a UV divergent part, which can be regulated and subracted as shown in the appendix A of [6]. The scattering length can then be extracted from eq. (2.1) using:

$$
a_{0}=\lim _{p \rightarrow 0} \frac{\tan \delta_{0}(p)}{p} .
$$

To evaluate this limit, we take $K$ and $\pi$ to be at rest, which results in the smallest possible value of $p$. The scattering length can then be taken to be equal to $\tan \delta_{0}(p) / p$ up to $\mathscr{O}\left(p^{2}\right)$ corrections. The problem is thus reduced to finding the accurate values of $E_{K \pi}, m_{K}$ and $m_{\pi}$ as described in the following sections. For comparison purposes, instead of calculating the scattering length itself, we find the dimensionless quantity $a_{0} m_{\pi}$. 


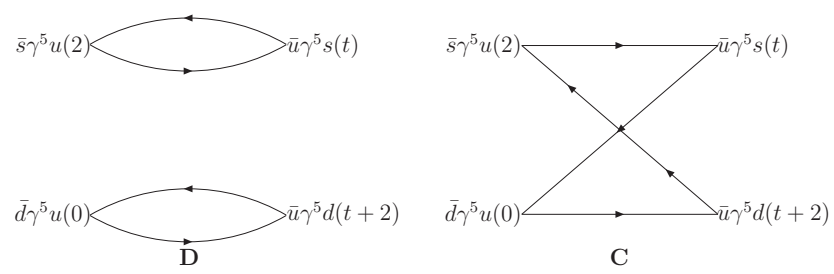

Figure 1: Contractions contributing to $K \pi$ scattering $\mathrm{I}=3 / 2$ channel.

\section{3. $K \pi$ scattering - contractions}

We can extract $K \pi$ energies (and hence $a_{0}$ ) from the following correlation function:

$$
C_{K \pi}^{I}(t) \equiv\left\langle O_{K \pi s n k}^{I}(t) O_{K \pi s r c}^{I}(0)\right\rangle,
$$

where $I$ denotes the isospin representation of the operator. We choose to use the two-meson interpolating operators, described below.

\section{1 $I=3 / 2$}

In the $I=3 / 2$ case, it's easiest to consider the highest weight state with $I_{3}=3 / 2$. This state consists of a single flavour eigenstate $K^{+} \pi^{+}$, which can be created by choosing:

$$
\begin{aligned}
& O_{K \pi s r c}^{3 / 2}(t)=T\left[\left(\bar{u} \gamma_{5} s\right)(t)\left(\bar{u} \gamma_{5} d\right)(t+\delta)\right], \\
& O_{K \pi s n k}^{3 / 2}(t)=T\left[\left(\bar{s} \gamma_{5} u\right)(t)\left(\bar{d} \gamma_{5} u\right)(t+\delta)\right],
\end{aligned}
$$

with $\delta= \pm 2$. We chose to split the meson in time to accomodate for stochastic $Z_{2} \times Z_{2}$ noise sources that we use for the propagators. This is done to avoid the additional, non-gauge invariant contractions that would arise if both operators were placed at the same time slice with the same noise vector. We chose to use only one noise source per time slice to keep the number of propagator inversions low. The contractions consist of two diagrams shown in figure 1 and are given by:

$$
\begin{aligned}
& D=\operatorname{Tr}\left(S^{\dagger}(t ; 2) L(t ; 2)\right) \operatorname{Tr}\left(L(t+2 ; 0)^{\dagger} L(t+2 ; 0)\right), \\
& C=\operatorname{Tr}\left(S^{\dagger}(t ; 2) L(2 ; 0) L^{\dagger}(t+2 ; 0) L(t+2 ; 2)\right),
\end{aligned}
$$

where $S\left(t_{f}, t_{i}\right)$ and $L\left(t_{f}, t_{i}\right)$ denote the strange and light quark propagators starting at time $t_{i}$ and ending at time $t_{f}$. The correlation function is found to be equal to:

$$
C_{K \pi}^{3 / 2}(t)=D-C
$$

\section{$3.2 \mathrm{I}=1 / 2$}

In the $\mathrm{I}=1 / 2$ case, the operator is a linear combination of three $I_{3}=1 / 2$ operators:

$$
O_{K \pi s r c}^{I=1 / 2}(t)=T\left[\alpha\left(\bar{d} \gamma_{5} s\right)\left(\bar{u} \gamma_{5} d\right)+\beta\left(\bar{u} \gamma_{5} s\right)\left(\bar{d} \gamma_{5} d\right)+\gamma\left(\bar{u} \gamma_{5} s\right)\left(\bar{u} \gamma_{5} u\right)\right]
$$



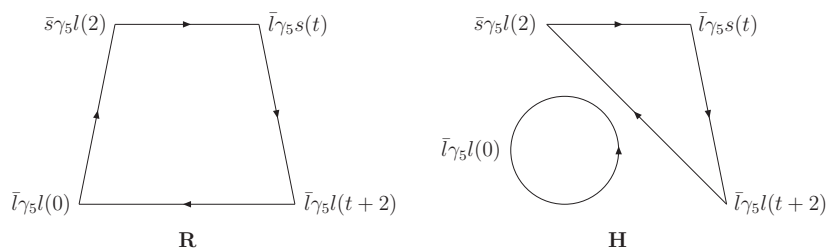

Figure 2: Additional contractions contributing to $K \pi$ scattering $\mathrm{I}=1 / 2$ channel, which we call $\mathrm{R}$ (left) and H(right).

For this operator to be in $\mathrm{I}=1 / 2$ representation, we must have $\alpha+\beta-\gamma=0$. Individually the contractions for each of these operators with $\left(\bar{s} \gamma_{5} d\right)\left(\bar{d} \gamma_{5} u\right)$ at the sink are:

$$
\begin{aligned}
& \left\langle\left[\left(\bar{s} \gamma_{5} d\right)\left(\bar{d} \gamma_{5} u\right)\right](t)\left[\left(\bar{d} \gamma_{5} s\right)\left(\bar{u} \gamma_{5} d\right)\right](0)\right\rangle=D-R, \\
& \left\langle\left[\left(\bar{s} \gamma_{5} d\right)\left(\bar{d} \gamma_{5} u\right)\right](t)\left[\left(\bar{u} \gamma_{5} s\right)\left(\bar{u} \gamma_{5} u\right)\right](0)\right\rangle=H-C, \\
& \left\langle\left[\left(\bar{s} \gamma_{5} d\right)\left(\bar{d} \gamma_{5} u\right)\right](t)\left[\left(\bar{u} \gamma_{5} s\right)\left(\bar{d} \gamma_{5} d\right)\right](0)\right\rangle=H-R,
\end{aligned}
$$

where the diagrams $\mathrm{D}$ and $\mathrm{C}$ are shown in Fig. 1 and the remaining two diagrams are in Fig. 2. The choice of the operator at the sink is immaterial, because the operator at the source will project it to the right isospin state. Our choice is $\alpha=1, \beta=\gamma=1 / 2$, which gives an operator which creates the $\mathrm{I}=1 / 2 K \pi$ state in the free theory and therefore eliminates all the self-contraction diagrams $(\mathrm{H}$ diagram). This combination is the most likely to have a good overlap with $\mathrm{I}=1 / 2 K \pi$ state. Other choices of $\alpha, \beta$ and $\gamma$ would correspond to different mixtures of $K \pi$ and $K \eta$ interpolating operators. We chose the same sources as in $\mathrm{I}=3 / 2$ case. While it may be beneficial to consider other source types and use the the generalised eigenvalue problem to separate the ground state $(\mathrm{K} \pi)$ from the first excited state $(\kappa)$, previous studies by other collaborations [5] showed that two-meson sources have good overlap with the ground state for light pion masses. The contractions can then be written as:

$$
R=\operatorname{Tr}\left(S^{\dagger}(t, 2) L(t, t+2) L^{\dagger}(0, t+2) L(0,2)\right) .
$$

and the correlation function becomes:

$$
C_{K \pi}^{1 / 2}(t)=D+0.5 C-1.5 R
$$

\section{4. $K \pi$ scattering - correlation functions}

Having calculated the correlation functions, we can use them to determine the energy of the $\mathrm{K}-\pi$ state. Taking one of the possible time orderings as an example, we have:

$$
\begin{aligned}
C_{K \pi}^{I}(t) & =\left\langle K^{\dagger}(t+2) \pi^{\dagger}(t) \pi(2) K(0)\right\rangle \\
& =|\langle K \pi|\pi(2) K(0)| 0\rangle|^{2} e^{-E_{K \pi}(t+2)} \\
& +|\langle 0|\pi(2) K(0)| K \pi\rangle|^{2} e^{-E_{K \pi}(T-t-2)} \\
& +|\langle K|\pi(2) K(0)| \pi\rangle|^{2} e^{-m_{\pi}(T-t-2)} e^{-m_{K}(t+2)} \\
& +|\langle\pi|\pi(2) K(0)| K\rangle|^{2} e^{-m_{K}(T-t-2)} e^{-m_{\pi}(t+2)} \\
& +\ldots
\end{aligned}
$$




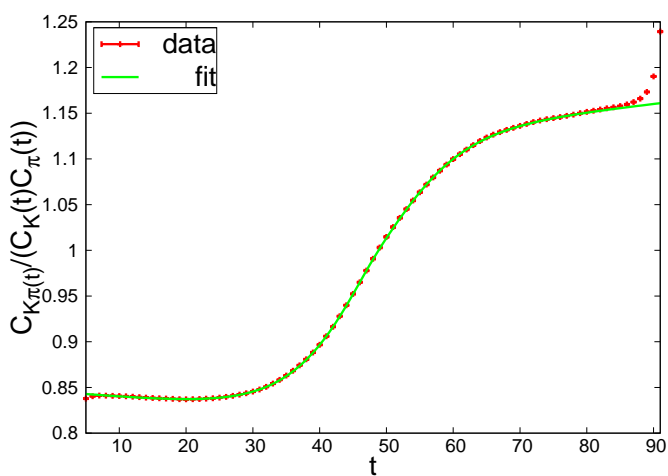

Figure 3: Ratio of $C_{K \pi}(t) /\left(C_{K}(t) C_{\pi}(t)\right)$ with the best fit curve.

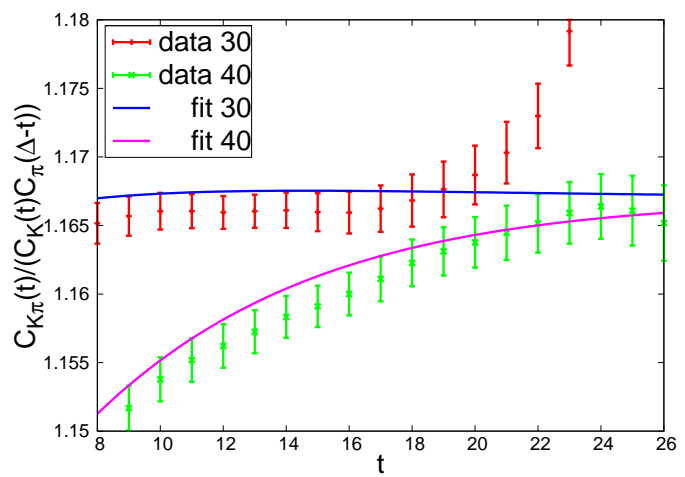

Figure 4: Fits for K-to- $\pi$ matrix elements with K-pi separation of 30 and 40.

In the above equations, the operator $\pi(2) K(0)$ is understood to be taken in the correct isospin projection - either $3 / 2$ or $1 / 2$. For sufficiently large time extent, $T$, contributions from the terms given by eqs. (4.3-4.5) will be negligible in comparison to the term given by eq. (4.2). In this case the correlation function has a time dependence given by a single exponential. Our experience with $\pi$ - $\pi$ scattering shows that the most accurate estimate of the ground state energy can be obtained by considering the ratio of the two-pion correlation function to the single pion two-point functions [7]. In $\mathrm{K}-\pi$ scattering case, the corresponding ratio would be:

$$
\frac{C_{K \pi}(t)}{C_{\pi}(t) C_{K}(t)} \approx A e^{-\Delta E t} \approx A(1-\Delta E t)
$$

where $\Delta E=E_{K \pi}-m_{K}-m_{\pi}$ and $\mathrm{A}$ is a constant. The second approximation in the above equations comes from assuming that $\Delta E$ is small. We therefore expect the ratio to be approximately linear for some $0 \ll t \ll T / 2$. An example of such ratio plot is shown in Fig. 3. We can see that the data points for the ratio do not match the single exponential behaviour. From this observation we can conclude that 'around-the-world' effects given by equations (4.3-4.5) should be included in the fitting procedure. This requires a five parameter fit, where the parameters are $E_{K \pi}$ and four normalization constants which appear in terms given by equations (4.2-4.5). Such fit turns out to be stable, but fitting five parameters simultaneously can result in a large statistical error. This issue can be dealt with by calculating the K-to- $\pi$ and $\pi$-to-K matrix elements in equations (4.4 and 4.5) from a different correlation function, which reduces the number of free parameters from 5 to 3 . The calculation of these matrix elements is described in the following section.

\section{Reducing the number of fitting parameters using $K$ to $\pi$ matrix elements}

As we mentioned in the previous section, and as shown in the results section below, we can improve the error by calculating the K-to- $\pi$ and $\pi$-to-K matrix elements in eqs. (4.4-4.5) separately. We outline the calculation of the K-to- $\pi$ matrix element below. The $\pi$-to-K matrix element can be 


\begin{tabular}{|l|l|l|}
\hline & $a_{0}^{3 / 2} m_{\pi}$ & $a_{0}^{1 / 2} m_{\pi}$ \\
\hline \hline NPLQCD & $-0.0574(16)\left(_{-58}^{+24}\right)$ & $0.1725(13)\left(_{-156}^{+23}\right)$ \\
Fu & $-0.0512(18)$ & $0.1819(35)$ \\
PACS-CS & $-0.0602(31)(26)$ & $0.183(18)(35)$ \\
\hline \hline this result & $-0.0674(33)$ & $0.1562(50)$ \\
\hline
\end{tabular}

Table 1: Comparison of our current result with previous lattice results. Where two error bars are given, the first corresponds to statistical error and the second to systematic error due to chiral extrapolation.

found in the same way. We can extract $K \rightarrow \pi$ matrix elements from:

$$
\begin{aligned}
C(t) & =\langle\pi(\Delta) \pi(t+2) K(t) K(0)\rangle \\
& =\langle 0|\pi| \pi\rangle\langle\pi|\pi(2) K(0)| K\rangle\langle K|K| 0\rangle e^{-m_{\pi}(\Delta-t)} e^{-m_{K} t} \\
& +\langle\pi|\pi| 0\rangle\langle 0|\pi(2) K(0)| K \pi\rangle\langle K \pi|K| \pi\rangle e^{-m_{\pi}(T-\Delta)} e^{-E_{K \pi} t} \\
& +\ldots
\end{aligned}
$$

with other contributions negligible in comparison. If there were no around-the-world effects, the ratio $C(t) /\left(C_{\pi}(\Delta-t) C_{K}(t)\right)=\langle\pi|\pi(2) K(0)| K\rangle /(\langle 0|\pi| \pi\rangle\langle K|K| 0\rangle)$ would be constant. This quantity is shown in Fig. 4. As before, the plot shows significant discrepancy from the above approximation, which suggests that the around-the-world effects can not be neglected. Examples of fits including the leading around-the-world contribution (eq. 5.3) are shown in figure 4. Fitted values for these correlation functions for the $\mathrm{K}-\pi$ matrix element in this figure are 1.1661(78) for $\mathrm{K}-\pi$ separation of 30 and 1.1697(34) for K- $\pi$ separation of 40 . We can see that, despite the apparent qualitative differences in correlation functions themselves, they are consistent to within $1 \sigma$.

\section{Results}

Our final values for scattering lengths using 3 and 5-parameter fits are:

\begin{tabular}{|l||l|l||l|l|}
\hline Number of parameters & $a_{0}^{3 / 2}$ & $\chi^{2} /$ dof & $a_{0}^{1 / 2}$ & $\chi^{2} /$ dof \\
\hline 5 & $-0.0638(73)$ & 0.0263 & $0.165(10)$ & 0.0805 \\
3 & $-0.0674(33)$ & 0.0307 & $0.1562(50)$ & 0.0805 \\
\hline
\end{tabular}

We can see that using a 3-parameter fit helps to reduce the error by about a factor of 2. A comparison with earlier lattice results is shown in table 1. A graphical comparison with lattice and phenomenological results [9] is in Fig. 5.

\section{Conclusions}

We have performed the first calculation of $\mathrm{K}-\pi$ scattering lengths directly at physical kinematics. Our results for $m_{\pi} a_{0}$ are -0.0674 (33) for $\mathrm{I}=3 / 2$ scattering and 0.1562(50) for $\mathrm{I}=1 / 2$ scattering, where the errors are purely statistical. The biggest limitation of the above calculation is that it is 


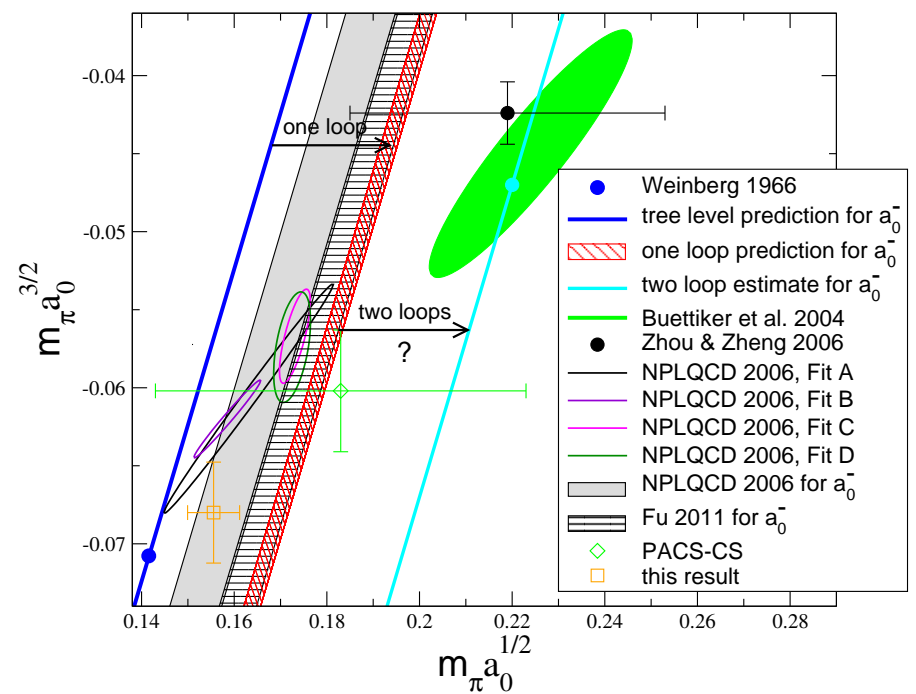

Figure 5: Comparison with current experimental and lattice results.

done using only a single lattice spacing, so we expect the error to be enhanced by $\mathscr{O}\left(a^{2}\right)$ effects. We will address this issue by repeating the calculation on an ensemble with a different lattice spacing and taking the continuum extrapolation in the near future.

Acknowledgements The research leading to these results has received funding from the European Research Council under the European Union's Seventh Framework Programme (FP7/20072013) / ERC Grant agreement 279757. The DiRAC equipment was funded by BIS National Einfrastructure capital grants ST/K000411/1, STFC capital grant ST/H008845/1, and STFC DiRAC Operations grants ST/K005804/1 and ST/K005790/1.

\section{References}

[1] M. Luscher, Nucl. Phys. B 354 (1991) 531.

[2] S. R. Beane, P. F. Bedaque, T. C. Luu, K. Orginos, E. Pallante, A. Parreno and M. J. Savage, Phys. Rev. D 74 (2006) 114503 [hep-lat/0607036].

[3] Z. Fu, JHEP 1201 (2012) 017 [arXiv:1110.5975 [hep-lat]].

[4] Z. Fu, Phys. Rev. D 85 (2012) 074501 [arXiv:1110.1422 [hep-lat]].

[5] K. Sasaki et al. [PACS-CS Collaboration], Phys. Rev. D 89 (2014) 054502 [arXiv:1311.7226 [hep-lat]].

[6] T. Yamazaki et al. [CP-PACS Collaboration], Phys. Rev. D 70 (2004) 074513 [hep-lat/0402025].

[7] T. Blum, P. A. Boyle, N. H. Christ, N. Garron, E. Goode, T. Izubuchi, C. Jung and C. Kelly et al., Phys. Rev. D 86 (2012) 074513 [arXiv:1206.5142 [hep-lat]].

[8] T. Blum et al. [RBC-UKQCD Collaboration], "Domain Wall QCD with Physical Quark Masses" [arXiv:1411.7017 [hep-lat]].

[9] P. Buettiker, S. Descotes-Genon and B. Moussallam, Eur. Phys. J. C 33 (2004) 409 [hep-ph/0310283]. 